\title{
Anthropometric profile and conditional factors of U21 Spanish elite beach volleyball players according to playing position \\ Perfil antropométrico y factores condicionales de los jugadores españoles élite de vóley playa sub- 21. según la posición de juego
}

Sergio Sebastia-Amat, Basilio Pueo, Lamberto Villalon-Gasch, Jose Manuel Jimenez-Olmedo

Universidad de Alicante (España)

\begin{abstract}
The aim of this work was to describe and study the relationship between anthropometric and conditional factors of under- 21 high-performance beach volleyball players according to playing position. The sample consisted of 5 male teams $(5$ blockers and 5 defenders) belonging to Spanish men's national beach volleyball team or participants in international tournaments. Anthropometric profile was assessed following the guidelines proposed by ISAK. The tests performed to assess conditional factors were: vertical jump (SJ, CMJ and ABK), 5- and 10-m sprint (S5m and S10m), agility test (AT) and overhead medicine ball throw in a standing position (OTSP) and on knees position (OTKP). Mann-Whitney U test was applied to compare blockers and defenders and Pearson correlation coefficient $(r)$ was used to determine the relationships between anthropometric and conditional variables. The results showed that U21 Spanish beach volleyball players had lower values for height and body weight than international players of the same category. Regarding playing position, blockers showed higher values of height, weight, muscle mass and bone weight than defenders ( $<<0.05)$. The somatotype for blockers and defenders were classified as ecto-mesomorph. Relationships have been found between anthropometric variables (height, weight, bone, muscle and fat) and conditional factors. The conditional tests did not show significant differences between blockers and defenders except those which required to mobilize an external weight, in which case blockers showed a better performance.
\end{abstract}

Keywords: sport performance, anthropometry, athletes, body composition, sport profile, somatotype.

Resumen: El objetivo del presente trabajo fue describir y estudiar la relación existente entre el perfil antropométrico y los factores condicionales de los jugadores de voleibol de playa de alto rendimiento en la categoría sub-21 en función de la posición de juego. La muestra consistió en 5 equipos ( 5 bloqueadores y 5 defensores) pertenecientes al equipo nacional de voleibol de playa masculino o participantes en torneos internacionales. El perfil antropométrico se evaluó siguiendo las pautas propuestas por ISAK. Las pruebas realizadas para evaluar los factores condicionales fueron: salto vertical (SJ, CMJ y ABK), sprint de 5 y $10 \mathrm{~m}$ (S5m y S10m), prueba de agilidad (AT) y lanzamiento de balón medicinal de pie (OTSP) y en posición de rodillas (OTKP). La prueba U de Mann-Whitney se aplicó para comparar los datos obtenidos de los bloqueadores y defensores, mientras que el coeficiente de correlación de Pearson $(r)$ se utilizó para determinar las relaciones entre las variables antropométricas y el rendimiento del juego. Los jugadores españoles sub-21 mostraron valores más bajos en variables como el peso y la altura cuando fueron comparados con jugadores internacionales de la misma categoría. Los bloqueadores presentaron valores más elevados en cuanto a la altura, peso, masa muscular y peso óseo que los defensores $(\mathrm{p}<0,05)$. El somatotipo tanto para los bloqueadores como para los defensores se clasificó como ecto-mesomorfo. Se encontraron relaciones entre las características antropométricas (altura, el peso, masa ósea, masa muscular y componente graso) y los factores condicionales. Las pruebas de rendimiento no mostraron diferencias significativas entre posiciones de juego, excepto en aquellas en las que se tuvo que movilizar una carga externa, en cuyo caso los bloqueadores mostraron mejores resultados.

Palabras clave: Rendimiento deportivo, antropometría, atletas, composición corporal, perfil deportivo, somatotipo.

\section{Introduction}

Beach volleyball is considered an intermittent sport that alternates brief periods of high intensity with longer periods of moderate and low intensity or pauses (Magalhães, Inácio, Oliveira, Ribeiro, \&Ascensão, 2011). The field measurements, game rules, number of players, instability of surface and weather conditions make it necessary to carry out beach volleyball skills at a high intensity to achieve success (Magalhães et al., 2011; Medeiros, Palao, Marcelino, \& Mesquita, 2014; Palao, Valadés, Manzanares, \& Ortega, 2014). Compared to volleyball indoor, more effort should be made to minimize the effect of force absorption by a sandy court and participate more actively in the game due to the smaller number of players (Koch \& Tilp, 2009). Others aspects, such as competitive level, playing position and age category influenced physical demands (Medeiros, Marcelino, Mesquita, \& Palao, 2014). Furthermore, physical demands

Fecha recepción: 27-11-19. Fecha de aceptación: 08-04-20 Basilio Pueo basilio@ua.es can also be affected by temporal characteristics like rally duration that increases the number of efforts exerted by players (Giatsis \& Papadopoulou, 2003).

Anthropometrical characteristics and motor performance variables are decisive factors for volleyball talent selection (Tsoukos et al., 2019). Several studies have reported that anthropometric characteristics and morphological parameters such as height, weight and percentage of body fat are correlated with higher beach volleyball performance both for senior (Palao, Gutierrez, \& Frideres, 2008) and junior categories (de Faria Pastore, de Azevedo Ferreira, da Costa, \& João, 2016; Quiroga, Sarmiento, Palomino, Rodriguez, \& Garcia, 2014). Some studies have reported differences in physical demands, anthropometric and technical-tactical characteristics when considering the player's role (JimenezOlmedo \& Penichet-Tomas, 2017; Jimenez-Olmedo, Pueo, Penichet-Tomás, Chinchilla Mira, \& Pérez Turpin, 2017), gender (Koch \& Tilp, 2009), level and age (Belem, Malheiros Caruzzo, Andrade do Nascimento Junior, Lopes Vieira, \& Fiorese Vieira, 2014; Medeiros, Marcelino, Mesquita, \& Palao, 2017).

Defensive tactics are the result of the coordination actions 
between blockers and diggers to defend the attack of the opponent (Jimenez-Olmedo \& Penichet-Tomas, 2017). The player's role in teams with defensive specialization is directly associated with different performance profiles although it does not happen the same with no defensive specialization (Kilary \& Shewman, 2000; Palao et al., 2008). According to the performance during the match, blockers may execute a higher number of jumps both to block every attack of the opponent and realize their own attack, so a high stature is important to perform both actions (Giatsis, Tili, \& Zetou, 2011; Medeiros et al., 2014). Conversely, defenders are usually shorter with a great capacity of displacement in sand, normally to defend the field zones that blockers leaves free or the balls that surpass the block (Natali, Ferioli, La Torre, \& Bonato, 2017; Schläppi-Lienhard \& Hossner, 2015). Moreover, there is a an alternative tactical system without defensive specialization, where both players block and dig. These players showed intermediate values of height compared to blockers and defenders (Palao et al., 2008) although physical demands compared to specialist players remain unknown. In view of this, Miliæ etal. (2017) highlighted the importance to know the anthropometric profile and conditional factors according to playing position and level of expertise.

Beach volleyball information is currently available about senior category. However, in recent years some studies have begun to evaluate performance capabilities in junior categories (de Faria Pastore et al., 2016; Medeiros et al., 2017; Medeiros et al., 2010) because of the impossibility of comparing data of different age categories (Medeiros, Marcelino, et al., 2014). In this way, Quiroga et al. (2014) described the anthropometrical profile of Spanish beach volleyball players in different age categories although they didn't report data about playing and ranking positions, and conditional factors. Thus, the aim of this work was to describe and study the relationship between anthropometric and conditional factors of under-21 high-performance beach volleyball players according to playing position.

\section{Material and methods}

\section{Subjects}

Five national sub-elite teams participated in the study, consisting of 10 male participants of high-performance beach volleyball players in the category under 21 belonging to national team (Spanish men's national beach volleyball team) or participants in international tournaments were selected: 5 blockers ( $19.8 \pm 1.3$ years) and 5 defenders $(19.0 \pm 1.2$ years $)$.

Measurements took place at the beginning of the competition period. The requirements to participate in the present study were: training regularly 5-7 times per week, 3 years competing in international tournaments and 2 years in the National Circuit «Madison Beach Volley Tour».

All the players were previously informed about the research aims, experimental protocol and procedures of the study and voluntary gave their informed written consent to participate. Ethical approval was obtained from the Institutional Ethics Board of the University of Alicante and the protocol was written in accordance with the standards established by the Declaration of Helsinki.

\section{Procedures}

\section{Anthropometric profile}

The International Society for the Advancement of Kinanthropometry (ISAK) protocol was used to determinate the anthropometric profile (Stewart, Marfell-Jones, Olds, \& Ridder, 2011) and the somatotype was determinate following Health-Carter's method (Carter, 1975). All measurements performed were measured by the same appraiser, certified anthropometrist Level 2 by the International Society for the Advancement of Kinanthropometry (ISAK). The technical measurement error was within the recommended ranges.

Weight was measured to the nearest $0.1 \mathrm{~kg}$ with a body composition analyzer (Tanita BC 545-N) and height using an stadiometer to the nearest $1 \mathrm{~mm}$. Skinfolds were measured using a calliper with a constant independent pressure of 10 $\mathrm{g} / \mathrm{mm}^{2}$ and reading to the nearest $1 \mathrm{~mm}$. Diameters were measured using an pachymeter to the nearest $1 \mathrm{~mm}$ and perimeters using a non-stretchable anthropometric tape to the nearest $1 \mathrm{~mm}$. All instruments were calibrated in advance to avoid errors in the measurement.

The registered variables were: height, weight, 8 Skinfolds (triceps, subscapularis, biceps, iliac crest, suprailiac, abdomen, thigh and leg), 3 diameters (bi-humeral, bi-femoral and by-styloid) and 6 perimeters (relaxed arm, contracted arm, waist, hip, thigh and leg). All measurements were carried out on the right side of the body.

Body composition was calculated from equations described in the consensus of kinanthropometry of GREC (Alvero et al., 2010) for male athletes. The equations used for this purpose were as follows: Fat weight by Carter's equation (1982), bone weight by Rocha's equation (1975) and muscle mass by Lee's equation (2000). Conditional tests were carried out in the sand except for vertical jump tests and $1 \mathrm{RM}$ tests. Two sessions of familiarization were held for all exercises, even though most of them are common tools in yearly training. Each player made 3 attempts with a rest of 3 minutes in order to avoid fatigue. The best attempt was used for the study.

Sprint performance in sand

Athletes performed two different types of sprint: 5-m and $10-\mathrm{m}$ sprint with three registration data points through photocells (Racetime2 Light radio; Microgate, Bolzano, Italy). Sprint time was recorded to the nearest 0.01 seconds. Testretest demonstrated high intra-session reliability of the 5-m sprint (ICC 0.902; CV 2.8) and 10-m sprint (ICC 0.916; CV 2.1).

Agility test in sand

T-test was administered from the proposal of Semenick (1990) with an ICC 0.846 and CV 2.5. T-test time was measured to the nearest 0.01 seconds.

\section{Vertical Jump}

Squat Jump (SJ), Countermovement Jump (CMJ) and Abalakov jump (ABK) were used to evaluate the jumping ability of beach volleyball players. For this purpose, a jump mat (Chronojump-Boscosystem, Barcelona, Spain) was used. The three types of jump showed a high intra-session reliability SJ (ICC 0.968; CV 3.2), CMJ (ICC 0.956; CV 2.8), ABK(ICC 0.977; CV 1.8).

\section{Overhead Medicine Ball Throw}

The explosive power was assessed with two types of medicine ball throws: Overhead medicine ball Throw from Standing Position (OTSP) (ICC 0.979; CV 2.6) and Overhead 
medicine ball Throw from Knees Position(OTKP) (ICC 0.989; CV 3.9). Athletes were instructed to throw a $5 \mathrm{~kg}$ medicine ball overhead as far forward as possible (Kawamori \& Haff, 2004). Throwing distance was measured to the nearest $1 \mathrm{~cm}$.

\section{$1 R M$ test}

One repetition maximum was determined according to procedures described by Kraemer \& Fry (1995). 1RM was calculated for bench press and half squat exercises. Each 1RM attempt was separated by 4 minutes of rest.

\section{Statistical Analyses}

Basic descriptive statistics by playing position (mean and standard deviation) were carried out for a description of the sample. Shapiro-Wilk test was applied to determine whether the quantitative variables fulfil the criteria of normality and $\mathrm{U}$ de Mann-Whitney for the comparison between blockers and defenders. The level of significance was set at 95\%. Effect size was calculated using Hedges' $\mathrm{g}$ formula for small samples. The effect size was defined as $<0.2$ trivial, $0.2-$ 0.6 small, $0.6-1.2$ moderate and $>1.2$ large. Pearson correlation coefficient $(r)$ was used to determine the relationships between anthropometric and conditional variables. Analyses were performed using the Statistical Package for Social Sciences (SPSS, v.24.0).

\section{Results}

Table 1 shows the anthropometric profile depending on the playing position. Blockers showed higher height and weight values than defenders $(p<0.05)$. The rest of the variables analyzed did not provide significant differences among playing position although some variables like waist perimeter tend to significance.

Table 1

\begin{tabular}{|c|c|c|c|c|c|c|}
\hline & \multicolumn{2}{|c|}{ Blockers (B) } & \multicolumn{2}{|c|}{ Defenders (D) } & \multirow{2}{*}{$\mathrm{p}$ value } & \multirow{2}{*}{$\begin{array}{c}\text { Effect } \\
\text { Size }\end{array}$} \\
\hline & $\mathrm{M}$ & SD & $\mathrm{M}$ & SD & & \\
\hline Height $(\mathrm{cm})$ & 190.82 & 6.22 & 180.36 & 5.27 & $0.021^{*}$ & 2.01 \\
\hline Weight (kg) & 83.98 & 8.75 & 72.70 & 6.18 & $0.048^{*}$ & 1.33 \\
\hline BMI $\left(\mathrm{kg} / \mathrm{m}^{2}\right)$ & 23.01 & 1.52 & 22.38 & 1.90 & 0.544 & 0.33 \\
\hline SF Triceps & 7.90 & 1.88 & 8.50 & 3.25 & 0.731 & -0.20 \\
\hline SF Subscapular & 8.70 & 2.38 & 8.90 & 1.71 & 0.883 & -0.09 \\
\hline SF Biceps & 4.10 & 0.74 & 4.60 & 0.65 & 0.319 & -0.65 \\
\hline SF Iliac crest & 10.70 & 3.27 & 12.69 & 5.04 & 0.478 & -0.42 \\
\hline SF Suprailiac & 7.10 & 1.43 & 8.56 & 2.53 & 0.295 & -0.64 \\
\hline SF Abdomen & 10.90 & 2.38 & 10.70 & 3.50 & 0.838 & 0.06 \\
\hline SF Thigh & 11.70 & 2.92 & 10.62 & 1.30 & 0.326 & 0.43 \\
\hline SF Leg & 7.50 & 1.09 & 5.80 & 0.83 & $0.012 *$ & 1.58 \\
\hline PR Relaxed arm & 32.38 & 1.78 & 30.82 & 2.54 & 0.179 & 0.64 \\
\hline PR Contracted arm & 34.32 & 1.96 & 32.24 & 2.47 & 0.293 & 0.84 \\
\hline PR Waist & 80.08 & 3.87 & 73.82 & 5.25 & 0.064 & 1.23 \\
\hline PR Hip & 97.72 & 3.78 & 94.84 & 3.08 & 0.223 & 0.75 \\
\hline PR Thigh & 57.88 & 1.25 & 56.48 & 2.92 & 0.354 & 0.56 \\
\hline PR Leg & 39.76 & 2.99 & 37.34 & 1.77 & 0.158 & 0.89 \\
\hline D. Bi-humeral & 7.26 & 0.36 & 7.00 & 0.23 & 0.217 & 0.78 \\
\hline D. Bi-femoral & 10.42 & 0.66 & 9.88 & 0.43 & 0.166 & 0.88 \\
\hline D. Bi- styloid & 6.02 & 0.15 & 5.90 & 0.12 & 0.201 & 0.80 \\
\hline
\end{tabular}

Values for body composition (fat, muscle and bone) were expressed in kilograms and percentage. The blockers presented higher values for all variables although results were only significant for bone mass variable (Table 2).

The somatotype graphic (Figure 1) shows that blockers and defenders had an ecto-mesomorph somatotype (2.01, $4.59,2.83)$ and $(2.28,4.38,3.14)$, respectively. Consequently, both playing position showed very similar somatotype, where mesomorphy was dominant and ectomorphy was higher than endomorphy.

Conditional variables are shown in Table 3. Comparison

\begin{tabular}{|c|c|c|c|c|c|c|}
\hline & \multicolumn{2}{|c|}{ Blockers (B) } & \multicolumn{2}{|c|}{ Defenders (D) } & \multirow{2}{*}{$\mathrm{p}$ value } & \multirow{2}{*}{$\begin{array}{l}\text { Effect } \\
\text { Size }\end{array}$} \\
\hline & $\mathrm{M}$ & SD & $\mathrm{M}$ & SD & & \\
\hline Muscle mass (kg) & 42.50 & 5.47 & 36.23 & 3.14 & 0.057 & 1.27 \\
\hline$\sum$ Muscle & 50.55 & 1.89 & 50.11 & 0.94 & 0.656 & 0.27 \\
\hline Bone mass $(\mathrm{kg})$ & 14.24 & 1.12 & 12.71 & 0.61 & $0.029 *$ & 1.53 \\
\hline$\sum$ Bone & 17.64 & 1.09 & 17.02 & 0.92 & 0.362 & -0.55 \\
\hline Fat mass (kg) & 6.96 & 0.92 & 5.93 & 1.37 & 0.200 & 0.80 \\
\hline$\sum$ Fat & 8.32 & 0.90 & 8.14 & 1.41 & 0.816 & 0.14 \\
\hline$\sum 6$ Skinfolds & 53.80 & 12.01 & 53.07 & 11.81 & 0.886 & 0.06 \\
\hline Endomorph & 2.01 & 0.48 & 2.28 & 0.67 & 0.393 & -0.53 \\
\hline Mesomorph & 4.59 & 1.42 & 4.38 & 1.33 & 0.810 & 0.14 \\
\hline Ectomorph & 2.83 & 1.16 & 3.14 & 1.08 & 0.669 & -0.25 \\
\hline
\end{tabular}

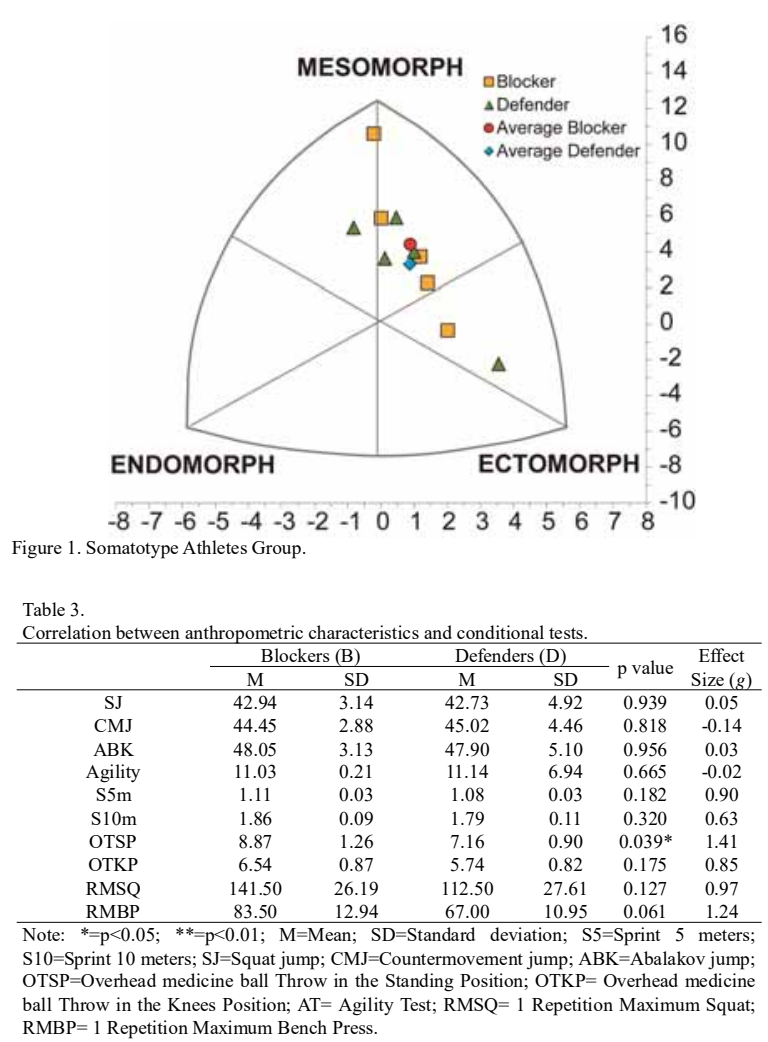

of blockers and defenders showed that differences were only significant for the variable of OTSP. The rest of variables showed no significance values although the variables in which an additional weight must be mobilized show a tendency to significance.

Table 4 show the correlation between anthropometric and conditional variables. The analyses of correlation showed that most of the anthropometric variables are correlated with each other, especially height with weight. A high correlation is also shown between diameters of the body and anthropometric characteristics, in particular with the diameter of the wrist. In the case of conditional variables, medicine ball throws (OTSP and OTKP) and RM tests (RMSQ and RMBP) were highly correlated with anthropometric variables. The rest of variables in which an additional weight was not necessary to be moved showed low correlation.

\section{Discussion}

There is little literature regarding the anthropometric and conditional factors of young beach volleyball players. Thus, the values of senior categories are usually taken as a reference despite being inappropriate due to the impossibility of comparing the data of different age categories (Medeiros et al., 2014). 


\begin{tabular}{|c|c|c|c|c|c|c|c|c|c|c|c|}
\hline & Position & $\mathrm{S} 5 \mathrm{~m}$ & S10m & SJ & CMJ & ABK & OTSP & OTSKP & AT & RMSQ & RMBP \\
\hline & A & 0.171 & 0.275 & 0.200 & 0.200 & 0.125 & $0.796^{*}$ & 0.799 ** & -0.219 & $0.665^{*}$ & $0.853^{* *}$ \\
\hline \multirow{3}{*}{ Height } & B & -0.589 & -0.043 & 0.471 & 0.538 & 0.193 & 0.789 & 0.841 & 0.108 & 0.456 & $0.904 *$ \\
\hline & D & 0.003 & 0.063 & 0.155 & 0.357 & 0.175 & 0.278 & 0.705 & $\begin{array}{l}-0.330 \\
-0.30\end{array}$ & 0.539 & 0.543 \\
\hline & $\mathrm{A}$ & 0.145 & 0.362 & 0.029 & -0.028 & 0.082 & $0.844^{* * *}$ & 0.625 & 0.156 & $0.765^{* *}$ & $0.935^{* * *}$ \\
\hline \multirow[t]{3}{*}{ Weight } & B & -0.800 & -0.193 & 0.348 & 0.379 & 0.609 & $0.989^{* *}$ & $0.992 * *$ & 0.129 & 0.864 & $0.986^{* *}$ \\
\hline & D & 0.480 & 0.626 & -0.284 & -0.264 & -0.335 & 0.181 & -0.285 & 0.607 & 0.415 & 0.755 \\
\hline & $\mathrm{A}$ & 0.074 & 0.290 & -0.170 & -.0248 & -0.018 & 0.525 & 0.200 & 0.459 & 0.531 & 0.613 \\
\hline \multirow[t]{3}{*}{ BMI } & B & -0.770 & -0.286 & 0.116 & 0.111 & 0.784 & $0.909 *$ & 0.869 & 0.155 & $0.989^{* *}$ & 0.800 \\
\hline & D & 0.401 & 0.495 & -0.324 & -0.393 & -0.376 & 0.053 & -0.529 & 0.671 & 0.111 & 0.405 \\
\hline & $\mathrm{A}$ & 0.317 & 0.517 & -0.151 & -0.134 & -0.306 & 0.542 & 0.362 & 0.393 & 0.445 & $0.803 * *$ \\
\hline \multirow[t]{3}{*}{ PR Relaxed arm } & B & -0.449 & -0.006 & 0.387 & 0.464 & -0.025 & 0.668 & 0.731 & 0.202 & 0.272 & 0.797 \\
\hline & D & 0.615 & 0.711 & -0.424 & -0.372 & -0.473 & 0.242 & -0.133 & 0.593 & 0.356 & 0.822 \\
\hline & $\mathrm{A}$ & 0.370 & 0.548 & -0.140 & -0.134 & -0.328 & 0.587 & 0.410 & 0.354 & 0.427 & $0.814^{* *}$ \\
\hline \multirow[t]{3}{*}{ PR Contracted arm } & B & -0.335 & 0.116 & 0.439 & 0.513 & -0.079 & 0.580 & 0.649 & 0.158 & 0.169 & 0.731 \\
\hline & D & 0.623 & 0.697 & -0.485 & -0.429 & -0.532 & 0.286 & -0.086 & 0.625 & 0.309 & 0.810 \\
\hline & $\mathrm{A}$ & 0.003 & 0.238 & 0.155 & 0.123 & 0.225 & $0.786^{* *}$ & 0.548 & 0.223 & $0.851^{* *}$ & $0.865^{* *}$ \\
\hline \multirow[t]{3}{*}{ PR Waist } & B & -0.784 & -0.125 & 0.424 & 0.433 & 0.784 & $0.948 *$ & $0.936 *$ & -0.027 & $0.927 *$ & $0.931 *$ \\
\hline & D & -0.107 & 0.134 & 0.056 & 0.116 & 0.034 & 0.405 & -0.047 & 0.555 & 0.704 & 0.702 \\
\hline & $\mathrm{A}$ & -0.152 & 0.199 & 0.240 & 0.226 & 0.365 & $0.745^{*}$ & 0.568 & 0.128 & $0.889^{* *}$ & $0.872^{* * *}$ \\
\hline \multirow[t]{3}{*}{ PR Hip } & B & -0.809 & -0.159 & 0.445 & 0.447 & 0.846 & $0.918^{*}$ & $0.901 *$ & -0.113 & $0.945^{*}$ & $0.899 *$ \\
\hline & D & 0.022 & 0.287 & 0.124 & 0.192 & 0.094 & 0.282 & -0.106 & 0.435 & 0.786 & 0.791 \\
\hline & $\mathrm{A}$ & -0.007 & 0.188 & 0.159 & 0.012 & 0.200 & 0.311 & -0.069 & 0.207 & 0.394 & 0.385 \\
\hline \multirow[t]{3}{*}{ PR Thigh } & B & $-0.879 *$ & -0.274 & 0.465 & 0.469 & 0.866 & $0.880 *$ & 0.864 & -0.218 & $0.936 *$ & 0.871 \\
\hline & D & 0.084 & 0.214 & 0.078 & -0.075 & 0.034 & -0.280 & -0.815 & 0.370 & 0.035 & -0.026 \\
\hline & $\mathrm{A}$ & 0.077 & 0.206 & -0.275 & -0.342 & -0.066 & $0.756^{*}$ & 0.583 & 0.259 & 0.545 & $0.730^{*}$ \\
\hline \multirow[t]{3}{*}{ PR Leg } & B & -0.786 & -0.398 & -0.039 & -0.016 & 0.551 & $0.959^{* *}$ & $0.934 *$ & 0.400 & $0.917 *$ & 0.851 \\
\hline & D & 0.794 & 0.674 & -0.725 & -0.789 & -0.771 & $\ldots 027$ & -0.352 & 0.536 & -0.402 & 0.207 \\
\hline & $\mathrm{A}$ & -0.193 & 0.033 & -0.062 & -0.086 & 0.003 & $0.879 * *$ & $0.707^{*}$ & 0.439 & 0.594 & $0.771^{* *}$ \\
\hline \multirow[t]{3}{*}{ D. Bi-humeral } & B & -0.663 & -0.105 & 0.239 & 0.279 & 0.421 & $0.958^{*}$ & $0.968^{* *}$ & 0.326 & 0.754 & $0.951 *$ \\
\hline & D & -0.244 & -0.197 & -0.416 & $\begin{array}{l}-0.402 \\
\end{array}$ & -0.414 & 0.705 & 0.113 & $0.924^{*}$ & 0.116 & 0.292 \\
\hline & $\mathrm{A}$ & 0.010 & 0.175 & -0.214 & -0.282 & -0.062 & $0.788^{* * *}$ & 0.557 & 0.397 & 0.572 & $0.754^{*}$ \\
\hline D. Bi- femoral & B & -0.738 & -0.314 & 0.007 & 0.039 & 0.465 & $0.971^{* *}$ & $0.959^{* *}$ & 0.442 & 0.856 & $0.891 *$ \\
\hline & D & 0.434 & 0.427 & -0.570 & -0.643 & -0.612 & 0.172 & -0.411 & 0.789 & -0.152 & 0.274 \\
\hline & $\mathrm{A}$ & 0.068 & 0.334 & 0.010 & 0.049 & 0.054 & $0.764^{*}$ & $0.734^{*}$ & 0.053 & $0.742^{*}$ & $0.959^{* *}$ \\
\hline D. Bi-styloid & B & -0.854 & -0.282 & 0.365 & 0.407 & 0.558 & $0.978^{* *}$ & $0.989 * *$ & 0.093 & 0.827 & $0.996^{* *}$ \\
\hline & D & 0.643 & 0.720 & -0.288 & -0.139 & -0.321 & 0.233 & 0.253 & 0.187 & 0.499 & $0.932 *$ \\
\hline & A & 0.113 & 0.379 & 0.132 & 0.099 & 0.153 & $0.789^{* *}$ & $0.662^{*}$ & -0.052 & $0.758^{*}$ & $0.953^{* *}$ \\
\hline Muscle mass $(\mathrm{kg})$ & B & -0.863 & -0.225 & 0.528 & 0.560 & 0.673 & $0.928 *$ & $0.940^{*}$ & -0.125 & 0.826 & $0.975^{* *}$ \\
\hline & D & 0.790 & $0.901 *$ & -0.240 & -0.170 & -0.296 & -0.030 & -0.163 & 0.221 & 0.442 & 0.866 \\
\hline & $\overline{\mathrm{A}}$ & -0.094 & 0.199 & 0.509 & 0.477 & 0.327 & 0.167 & 0.211 & -0.563 & 0.203 & 0.368 \\
\hline$\sum$ Muscle & B & -0.552 & -0.352 & -0.324 & -0.294 & 0.237 & 0.827 & 0.804 & 0.715 & 0.716 & 0.685 \\
\hline & D & 0.566 & 0.518 & 0.253 & 0.175 & 0.221 & $\ldots$ & -0.474 & -0.744 & -0.227 & $\begin{array}{r}-0.238 \\
\end{array}$ \\
\hline & $\mathrm{A}$ & 0.238 & 0.272 & -0.216 & -0.236 & -0.040 & $0.824 * *$ & $0.729^{*}$ & 0.145 & $0.702^{*}$ & $0.838^{* * *}$ \\
\hline $\begin{array}{l}\text { Bone } \\
\text { mass }(\mathrm{kg})\end{array}$ & B & 0.666 & 0.012 & -0.856 & -0.864 & -0.729 & -0.565 & -0.586 & 0.659 & -0.562 & -0.701 \\
\hline mass $(\mathrm{kg})$ & $\mathrm{D}$ & 0.647 & 0.664 & -0.422 & -0.265 & -0.446 & 0.322 & 0.372 & 0.232 & 0.363 & 0.867 \\
\hline & $\mathrm{A}$ & -0.046 & -0.413 & -0.277 & -0.246 & -0.185 & -0.460 & -0.261 & -0.014 & -0.583 & $-0.730^{*}$ \\
\hline$\sum$ Bone & B & 0.666 & 0.012 & -0.856 & -0.864 & -0.729 & -0.565 & -0.586 & 0.659 & -0.562 & -0.701 \\
\hline & D & -0.513 & -0.694 & 0.127 & 0.129 & 0.186 & 0.013 & 0.442 & -0.455 & -0.467 & -0.723 \\
\hline & $\mathrm{A}$ & 0.205 & 0.297 & -0.274 & -0.286 & -0.094 & 0.626 & 0.375 & 0.543 & $0.666^{*}$ & $0.686^{*}$ \\
\hline Fat mass $(\mathrm{kg})$ & B & -0.278 & -0.151 & -0.441 & -0.458 & 0.332 & 0.593 & 0.536 & 0.667 & 0.660 & 0.382 \\
\hline & D & 0.187 & 0.348 & -0.268 & -0.201 & -0.298 & 0.473 & -0.008 & 0.713 & 0.525 & 0.788 \\
\hline & $\mathrm{A}$ & 0.161 & 0.116 & -0.402 & -0.388 & -0.251 & 0.200 & -0.036 & 0.732 & 0.264 & 0.186 \\
\hline$\sum$ Fat & B & 0.434 & 0.066 & -0.783 & -0.826 & -0.214 & -0.197 & -0.258 & 0.674 & -0.055 & -0.420 \\
\hline & D & -0.043 & 0.108 & -0.251 & -0.201 & -0.269 & 0.572 & 0.028 & 0.793 & 0.456 & 0.630 \\
\hline & $\mathrm{A}$ & -0.219 & $-0.664^{*}$ & -0.629 & -0.631 & -0.415 & 0.023 & 0.017 & 0.490 & -0.377 & -0.449 \\
\hline$\sum 6$ Skinfolds & B & 0.177 & -0.553 & $-0.973 * *$ & $-0.968^{* *}$ & -0.504 & -0.323 & -0.361 & 0.632 & -0.229 & -0.510 \\
\hline & D & -0.604 & -0.808 & -0.435 & -0.450 & -0.378 & 0.630 & 0.472 & 0.479 & -0.577 & -0.552 \\
\hline & $\mathrm{A}$ & -0.062 & -0.062 & -0.417 & -0.398 & -0.290 & -0.033 & -0.221 & $0.827 * *$ & -0.050 & -0.109 \\
\hline Endomorphy & B & 0.378 & 0.144 & -0.709 & -0.732 & -0.258 & -0.008 & -0.052 & 0.813 & 0.005 & -0.204 \\
\hline & D & -0.111 & -0.006 & -0.301 & -0.314 & -0.317 & 0.503 & -0.130 & 0.859 & 0.219 & 0.368 \\
\hline & $\mathrm{A}$ & -0.182 & 0.102 & -0.102 & -0.155 & -.0090 & 0.500 & 0.274 & 0.439 & 0.294 & 0.535 \\
\hline Mesomorphy & B & -0.842 & -0.247 & 0.431 & 0.475 & 0.551 & $0.958^{*}$ & $0.975^{* *}$ & 0.034 & 0.793 & $0.998^{* *}$ \\
\hline & D & 0.370 & 0.366 & -0.477 & -0.587 & .0519 & 0.048 & -0.542 & 0.718 & -0.218 & 0.121 \\
\hline & $\mathrm{A}$ & 0.120 & -0.217 & -0.111 & -0.048 & -0.088 & -0.417 & -0.172 & -0.213 & -0.397 & -0.563 \\
\hline Ectomorphy & B & 0.829 & 0.188 & -0.702 & -0.722 & -0.752 & -0.762 & -0.775 & 0.441 & -0.735 & -0.850 \\
\hline & D & -0.432 & -.0507 & 0.296 & 0.396 & 0.350 & 0.081 & 0.644 & -0.596 & -0.006 & -0.278 \\
\hline
\end{tabular}

${ }^{*}=\mathrm{p}<0.05 ;{ }^{* *}=\mathrm{p}<0.01 ; \mathrm{A}=$ average values; B= Blockers, D=Defenders; M=Mean; BMI=Body mass index. SF=Skinfold. PR=Perimeter. Di=Diameter; SD=Standard deviation; S5=Sprint $5 \mathrm{~m} ; \mathrm{S} 10=\mathrm{Sprint} 10$

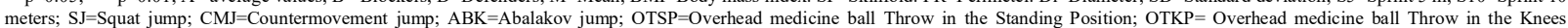
meters; $\mathrm{S}=\mathrm{Squat}$ jump; $\mathrm{CMJ}=\mathrm{Countermovement}$ jump; $\mathrm{ABK}=\mathrm{Abalakov}$ jump; $\mathrm{OTSP}=$ Overhead medicine ball Throw in the Standing Position; $\mathrm{O}$
Position; $\mathrm{AT}=$ Agility Test; $\mathrm{RMSQ}=1$ Repetition Maximum Squat; RMBP=1 Repetition Maximum Bench Press; $\mathrm{A}=\mathrm{All}$ players; $\mathrm{B}=\mathrm{Blockers;}$ Defenders.

Noori \& Sadeghi (2017) showed in their model for volleyball talent identification the importance of anthropometrical and conditional factors. Height and upper extremity length (anthropometrics), agility and power (biomechanics) and special endurance (aerobic and anaerobic) were the main variables related to volleyball players success.Similarly, Quiroga et al. (2014) reported the relevance of the anthropometrical profile to know the performance factors in sports and also to specialize the training program according to age category and playing position.

In beach volleyball, height is an important characteristic since most decisive actions in male category occur above a net located at $243 \mathrm{~cm}$ high. The results of the present study $(186 \mathrm{~cm})$ showed higher average $(A)$ height values to those observed by Quiroga et al. (2014) for the same category (183 $\mathrm{cm}$ ) (Quiroga et al., 2014). This may be due to the fact that our sample consisted only of high-level players. Converserly, the results were lower compared to U21 world championship 2017 participants $(191 \mathrm{~cm})(\mathrm{FIVB}, 2017)$ and senior category (190-194 cm) (Giatsis et al., 2011; Palao et al., 2008).

Regarding playing position, blockers showed larger height values than defenders $(191 \mathrm{~cm}, 180 \mathrm{~cm}$, difference $=11$ $\mathrm{cm})$. Similar results were found in U21 world championship 2017 participants $(197 \mathrm{~cm}, 185 \mathrm{~cm}$, difference $=11 \mathrm{~cm})$. Nevertheless, smaller differences were found between blockers and defenders height in senior category $(197 \mathrm{~cm}$, $190 \mathrm{~cm}$, difference $=7 \mathrm{~cm}$ ) (Palao et al., 2008), suggesting that the differences between blockers and defenders are smaller as level and age increases. These results coincide with those obtained by Giatsis et al. (2011) where concluded that blocking is very important in the course of game. Consequently, the height could be the main factor in beach volleyball players, especially in blockers.

Other important factors to consider are weight and 
percentage of body fat due to its relation with jump performance (Pérez-López, Sinovas, Álvarez-Valverde, \& Valades, $2015)$. In the present study, the weight values $(A=78,5 \mathrm{~kg}, B$ $=84 \mathrm{~kg}$ and $\mathrm{D}=73 \mathrm{~kg})$, were lower compared to international senior category $(A=89 \mathrm{~kg}, \mathrm{~B}=92 \mathrm{~kg}$ and $\mathrm{D}=86 \mathrm{~kg}$ ) (Palao et al., 2008) and U21 world championship 2017 participants (A= $82 \mathrm{~kg}, \mathrm{~B}=92 \mathrm{~kg}$ and $\mathrm{D}=86 \mathrm{~kg}$ ) (FIVB, 2017). Comparing our results to other studies carried out with Spanish beach volleyball players, Quiroga et al. (2014) found lower height and weight values for the same category $(<2.70 \mathrm{~cm}, 2.69 \mathrm{~kg})$ and higher values for Spanish senior category $(>1.34 \mathrm{~cm}, 4.9$ $\mathrm{kg}$ ), although this study didn't distinguish between playing position.

Beach volleyball players as volleyball indoor players have a low percentage of body fat regardless playing position. This fact could be observed in the study of Garrido-Chamorro, Sirvent-Belando, González-Lorenzo, Blasco-Lafarga, \& Roche (2012) in which elite athletes of different disciplines were compared. The results of body fat percentage found in the present study ( $8.32 \%$ for blockers and $8.14 \%$ for defenders) were similar to the results reported for the same category (8.4\% - 9.16\%) (Quiroga et al., 2014). Considering player position in junior categories, blockers have higher values of body fat than defenders (de Faria Pastore et al., 2016). This fact also occurs in the present study although the diference was non-significant.

The somatotype of players influence sports performance (Mielgo-Ayuso, Calleja-González, Clemente-Suárez, \& Zourdos, 2015). Consistent with the above, the results of the present study showed that high values of endomorphy seem to impair performance in jump and displacement skills. Conversely, high values of mesomorphy seem to facilitate a greater force production for displacing an external load.

The somatotype of the players revealed similar profile regardless of playing position. Blockers and defenders were classified as ecto-mesomorph. These results are similar to those found by Quiroga et al. (2014) for U21 beach volleyball players although the values of mesomorphy were lower to our own. On the other hand, Martínez \& Sanz (2012) reported a meso-endomorph somatotype in university players of the same age. The fact that the athletes of this study showed larger mesomorphic values may be due to physical characteristics of the Spanish population, talent selection process, the way of training in young categories and competitive level.

A remarkable correlation was found between bone weight and bone diameters, particularly the bi-styloid diameter, with some conditional variables. These results make sense since the radiographic evaluation of hand-wrist is used to determinate skeletal maturation(Chapman, 1972). Comparing the results obtained with others studies, Quiroga et al. (2014) reported lower values for the same category $(5.88 \mathrm{~cm})$ and similar values for senior category $(5.97 \mathrm{~cm})$ which may be due to the fact that participants in the present study could have an advanced maturation status.

The anthropometric variables that showed the highest correlation with conditional factors were height and weight, especially with conditional variables in which an external weight to the body had to be mobilized (overhead throws tests and RM tests) (Caruso et al., 2012). In this sense,
D'Isanto, Di Tore, \&Altavilla (2018) reported similar results, in which anthropometric characteristics like body mass and height contribute to athletes' success in volleyball.

Conditional variables showed that there were no significant differences between blockers and defenders performance except for OTSP (19.27\%). Besides, the variables that tended to show significant differences between playing positions were tests where athletes mobilized an external weight. Therefore, it is reasonable that blockers showed better values in these type of tests due to physical characteristics. Similarly, blockers showed stronger correlations between anthropometric characteristics and conditional tests than defenders possibly due to the greater physical demands required in blocker position and the advantages of blocker's anthropometrical profile for beach volleyball skills (Palao et al., 2014).

In view of the results, it does not seem logical to keep the idea that defenders must be shorter than blockers with the purpose of having a better displacement on the court during defensive actions. In fact, blockers showed similar or even better results for conditional variables.

\section{Conclusion}

U21 Spanish beach volleyball players had lower values of height and body weight than international players of the same cathegory. Considering playing position, blockers showed higher height and weight values than defenders. In the same way that volleyball indoor, beach volleyball players showed a low percentage of body fat regardless of the playing position. Likewise, these players showed an ectomesomorph somatotype both for blockers and defenders.

The conditional tests did not show significant differences between blockers and defenders except those which required to mobilize an external weight, in which case blockers showed a better performance.

The information showed in the present study offers reference values for beach volleyball coaches when it comes to carrying out the talent selection process.

\section{Acknowledgements}

The authors would like to thank all the participants in this study. Sergio Sebastiá-Amat participated in this study supported by a pre-doctoral grant (ACIF/2018/209) from the Generalitat Valenciana, Spain.

\section{References}

Alvero, J., Cabañas, M., Herrero de Lucas, A., Martínez, L., Moreno, C., Porta, J., ... Sirvent, J. E. (2010). Protocolo de valoración de la composición corporal para el reconocimiento médico-deportivo. Documento de consenso del Grupo Español de Cineantropometría (GREC) de la Federación Española de Medicina del Deporte (FEMEDE). Versión 2010. Archivos de Medicina Del Deporte, 27(139), 330-346.

Belem, I. C., Malheiros Caruzzo, N., Andrade do Nascimento Junior, J. R., Lopes Vieira, J. L., \& Fiorese Vieira, L. (2014). Impact of coping strategies on resilience of elite beach volleyball athletes. Revista Brasileira de Cineantropometria \& Desempenho Humano, 16(4), 447-455. https://doi.org/10.5007/1980-0037.2014v16n4p447

Carter, J. (1975). The Heath-Carter somatotype method. San Diego, 
California: San Diego State University.

Carter, J. E. . (1982). Body composition of Montreal Olympic athletes. In Physical structure of Olympic athletes Part I The Montreal Olympic Games Anthropological Project (pp. 107-116). Basel, Switzerland: Karger.

Caruso, J. F., Taylor, S. T., Lutz, B. M., Olson, N. M., Mason, M. L., Borgsmiller, J.A., \& Riner, R. D. (2012). Anthropometry as a predictor of bench press performance done at different loads. The Journal of Strength \& Conditioning Research, 26(9), 2460-2467. https://doi.org/ 10.1519/JSC.0b013e31823c44bb

Chapman, S. M. (1972). Ossification of the adductor sesamoid and the adolescent growth spurt. The Angle Orthodontist, 42(3), 236-244.

D'Isanto, T., Di Tore, P. A., \& Altavilla, G. (2018). Correlation of the anthropometric characteristics and the ability to jump in volleyball. Journal of Human Sport and Exercise, 13, S393-S400. https://doi.org/ 10.14198/jhse.2018.13.Proc2.23

de Faria Pastore, J. C., de Azevedo Ferreira, C. A., da Costa, F. C. H., \& João, P. (2016). Kinanthropometric Profile of Beach Volleyball Player of Category under 19 and under 21 of European Championship 2015 Kinanthropometric Profile of Beach Volleyball Player of. International Journal of Sports and Physical Education, 2(2), 20 24.

FIVB. (2017). FIVB Beach Volleyball U21 world Championships. Retrieved from http://u21.2017.beachvolleyball.fivb.com/en/men

Garrido-Chamorro, R., Sirvent-Belando, J. E., González-Lorenzo, M., Blasco-Lafarga, C., \& Roche, E. (2012). Skinfold Sum: Reference Values for Top Athletes Sumatorio de Pliegues Subcutáneos: Valores de Referencia para Atletas de Élite. Int. J. Morphol, 30(3), 803-809. https://doi.org/10.4067/S0717-95022012000300005

Giatsis, G. \& Papadopoulou, S. (2003). Effects of reduction in dimensions of the court on timing characteristics for men's beach volleyball matches. International Journal of Volleyball Research, 6(1), 7-10.

Giatsis, G, Tili, M., \& Zetou, E. (2011). The height of the men's winners FIVB Beach Volleyball in relation to specialization and court dimensions. Journal of Human Sport and Exercise, 6(3), 497-503. https://doi.org/10.4100/jhse.2011.63.03

Jimenez-Olmedo, J. M., \& Penichet-Tomas, A. (2017). Blocker's activity at men 's european beach volleyball university championship Actividad de los bloqueadores durante el Campeonato de Europa Universitario de vóley playa. Retos. Nuevas Tendencias En Educación Física, Deporte y Recreación, 32, 252-255.

Jimenez-Olmedo, J. M., Pueo, B., Penichet-Tomás, A., Chinchilla Mira, J. J., \& Pérez Turpin, J. A. (2017). Physiological work areas in professional beach volleyball: A case study. Retos. Nuevas Tendencias En Educación Fisica, Deporte y Recreación, (31), 94-97.

Kawamori, N., \& Haff, G. G. (2004). The optimal training load for the development of muscular power. Journal of Strength and Conditioning Research, 18(3), 675-684.

Kilary, K., \& Shewman, B. (2000). Vóley playa, técnicas, entrenamiento y tácticas del mejor jugador del mundo. Barcelona: Paidotribo.

Koch, C., \& Tilp, M. (2009). Beach volleyball: Techniques and tactics. A comparison of male and female playing characteristics. Kinesiology, 41(1), 52-59.

Kraemer, W., \& Fry, A. (1995). Development and Evaluation of Methodology. Champaign, IL: Human Kinetics.

Lee, R., Wang, Z., Heo, M., Ross, R., Janssen, I., \& Heymsfield, S. (2000) Total-body skeletal muscle mass: development and cross-validation of anthropometric prediction models. The American Journal of Clinical Nutrition, 72(3), 796-803. https://doi.org/10.1093/ajcn/ 72.3 .796

Magalhães, J., Inácio, M., Oliveira, E., Ribeiro, J. C., \& Ascensão, A (2011). Physiological and neuromuscular impact of beach-volleyball with reference to fatigue and recovery. Journal of Sports Medicine and Physical Fitness, 51(1), 66-73.

Martínez-, \& Sanz. (2012). Estudio de la composición corporal en deportistas masculinos universitarios de difertentes disciplinas deportivas. Cuadernos de Psicología Del Deporte, 12(1), 89-94.

Medeiros, A. I., Marcelino, R., Mesquita, I. M., \& Palao, J. M. (2017) Performance differences between winning and losing under-19, under21 and senior teams in men's beach volleyball. International Journal of Performance Analysis in Sport, 17(1-2), 96-108. https://doi.org/ 10.1080/24748668.2017.1304029

Medeiros, A., Marcelino, R., Mesquita, I., \& Palao, J. M. (2014). Physical and temporal characteristics of under 19, under 21 and senior male beach volleyball players. Journal Os Sports Science and Medicine, 13(3), 658-665.

Medeiros, A., Mesquita, I., Oliveira, J., Loureiro, A. C. C., Afonso, J., Monteiro, L. Z., \& Castro, J. M. (2010). Body composition of Brazilian beach volleyball players. British Journal of Sports Medicine, 44(14), i17. http://dx.doi.org/10.1136/bjsm.2010.078972.51

Medeiros, A., Palao, J. M., Marcelino, R., \& Mesquita, I. (2014). Systematic review on sports performance in beach volleyball from match analysis. Revista Brasileira de Cineantropometria e Desempenho Humano, 16(6), 698-709. http://dx.doi.org/10.5007/19800037.2014v16n6p698

Mielgo-Ayuso, J., Calleja-González, J., Clemente-Suárez, V. J., \& Zourdos, M. C. (2015). Influence of anthropometric profile on physical performance in elite female volleyballers in relation to playing position. Nutricion Hospitalaria, 31(2), 849-857. https://doi.org/10.3305/ nh.2015.31.2.7658

Miliæ, M., Grgantov, Z., Chamari, K.,Ardigò, L. P., Bianco, A., \& Padulo, J. (2017). Anthropometric and physical characteristics allow differentiation of young female volleyball players according to playing position and level of expertise. Biology of Sport, 34(1), 19-26. https://doi.org/10.5114/biolsport.2017.63382

Natali, S., Ferioli, D., La Torre, A., \& Bonato, M. (2017). Physical and technical demands of elite beach volleyball according to playing position and gender. The Journal of Sports Medicine and Physical Fitness, 59(1), 6-9. https://doi.org/10.23736/S0022-4707.17.079725

Noori, M., \& Sadeghi, H. (2017). Designing smart model in volleyball talent identification via fuzzy logic based on main and weighted criteria resulted from the analytic hierarchy process. Journal of Advanced Sport Technology, 1(2), 16-24.

Palao, J. M., Gutierrez, D., \& Frideres, J. E. (2008). Height, weight, Body Mass Index, and age in beach volleyball players in relation to level and position. J Sports Med Phys Fitness, 48(4), 466-471.

Palao, J. M., Valadés, D., Manzanares, P., \& Ortega, E. (2014). Physical actions and work-rest time in men's beach volleyball. Motriz: Revista de Educação Física, 20(3), 257-261. https://doi.org/10.1590/S198065742014000300003

Pérez-López, A., Sinovas, M. C., Álvarez-Valverde, I., \& Valades, D. (2015). Relationship between body composition and vertical jump performance in young spanish soccer players. Journal of Sport and Human Performance, 3(3), 1-12. https://doi.org/10.12922/ jshp.0063.2015

Quiroga, M. E., Sarmiento, S., Palomino, A., Rodriguez, D., \& Garcia, J. M. (2014). Anthropometric characteristics of spanish beach volleyball players. comparison by categories. International Journal of Morphology, 32(1), 22-28.

Rocha, M.S, L. (1975). «Peso osseo do brasileiro de ambos os sexos de 17 a 25 anos». Arquivos de Anatomia e Antropologia, 1, 445-451.

Schläppi-Lienhard, O., \& Hossner, E. J. (2015). Decision making in beach volleyball defense: Crucial factors derived from interviews with top-level experts. Psychology of Sport and Exercise, 16, 60-73. https://doi.org/10.1016/j.psychsport.2014.07.005

Semenick, D. (1990). Tests and measurements: The t-test. Strength \& Conditioning Journal, 12(1), 36-37.

Stewart,A., Marfell-Jones, M., Olds, T., \& Ridder, H. I. (2011). International Standards for anthropometric assessment. International Society for the Advancement of Kinanthropometry (ISAK). Lower Hutt, New Zealand: International Society for the Advancement of Kinanthropometry.

Tsoukos, A., Drikos, S., Brown, L. E., Sotiropoulos, K., Veligekas, P., \& Bogdanis, G. C. (2019). Anthropometric and Motor Performance Variables are Decisive Factors for the Selection of Junior National Female Volleyball Players Selection of Junior National Female Volleyball Players. Journal of Human Kinetics, 67(1), 163-173. https://doi.org/10.2478/hukin-2019-0012 